\title{
CHAOS AND STRANGE ATTRACTORS IN PULSAR INTENSITY RECORDS
}

\author{
ROger W. Romani \\ Institute for Advanced Study, Princeton Universuty \\ J. M. RANKIN \\ Department of Physics, University of Vermont \\ D. C. BACKER \\ Radio Astronomy Laboratory, University of California
}

\begin{abstract}
We have examined high quality Arecibo data of three pulsars for evidence of strange attractors in pulse-to-pulse intensity fluctuations. Significant structure was found for PSR $0823+26$, and seems related to pulse drifting and nulling activity. The low dimensionality of the possible attractor suggest that a simple dynamical model can explain this component of the flux variability.
\end{abstract}

\section{Introduction}

In addition to their simple regular pulsation, radio pulsars show a wealth of temporal variation in their emitted radio flux. On short time scales the pulses are modulated by 'microstructure' $(\tau \lesssim 100 \mu \mathrm{s})$ and subpulses $(\tau \sim 10 \mathrm{~ms})$, which provide varying illumination of components within the integrated pulse profile. Drifting subpulses, pulse nulling and mode changing give rise to intensity fluctuations between individual pulses, and in some cases cause correlated modulations extending for some hundreds of periods. For longer times, the integrated pulse profile of most pulsars are very stable. Slow modulation of the pulsar intensity due to diffractive $(\tau \gtrsim 100 \mathrm{~s})$ and refractive ( $\tau \sim$ days to years) scintillation are extrinsic, being caused by propagation through the ionized interstellar medium.

The poorly understood mechanism by which radio pulsars produce their coherent emission should leave its imprint on the intensity records. Dynamics of the plasma in the pulsar emission zone should cause microstructure-like variations on light crossing times, while the existence of correlated behavior such as drifting subpulses suggest that coherent dynamical structures in the pulsar magnetosphere can last for $\sim 10 \mathrm{~s}$. By characterizing the temporal fluctuations of the light curves on these time scales, we hope to derive constraints on the underlying physical mechanism.

The same path through the magnetosphere is viewed once per pulsar period; thus, to isolate temporal variations, we concentrate on pulse-to-pulse modulations. Except for pulsars with well defined drifting subpulses, power spectra generally show little structure at these frequencies; autocorrelation functions, however, show significant power at lags of a few pulses. In some cases this power may be chaotic, i.e. the result of some deterministic process in the system rather than purely stochastic. The study of such behavior, deterministic chaos, has attracted much attention in a number of scientific disciplines; in astrophysics researchers have looked for chaotic processes in the light curves of X-ray binaries (Voges, Atmanspacher, and Scheingraber 1987, Lochner, Swank, and Szymkowiak 1989) and in the arrival time residuals of radio pulsars (Harding, Shinbrot, and Cordes 1989). We were directed towards the study of radio pulsar flux variations by the observation of Backer (1971) that histograms of pulse intensity for some pulsars follow the form of chi-square distributions with few degrees of freedom. The presence of chaos, as evidenced by attractors in such data, allows one to infer that the fluctuations are being controlled by a process with a limited number of degrees of freedom. When the dimensionality of the attractor is low, it is argued that this may motivate simple models for the underlying physical process.

\section{Observations and correlation analysis}

High time resolution measurements of three strong pulsars were made with the Arecibo $305-\mathrm{m}$ telescope are listed in table 1 ( $c f$. Rankin, Stinebring and Weisberg 1989, hereafter RSW).

The integrated pulse profiles of these pulsars show substantial structure at $1.4 \mathrm{GHz}$. For PSR 0823+26 the observation covers the main pulse, which displays a single strong component. This was classified by RSW as $S_{t}$ (i.e. single core component) emission, although the presence of a postcursor some $35^{\circ}$ away and some ordered subpulse behavior made this identification uncertain. 
Table 1 Observations of bright pulsars

\begin{tabular}{|ccccccr|}
\hline Pulsar & $\begin{array}{c}\mathrm{P} \\
(\mathrm{s})\end{array}$ & $\begin{array}{c}\text { BW } \\
(\mathrm{MHz})\end{array}$ & $\begin{array}{c}t_{\text {samp }} \\
(\mathrm{ms})\end{array}$ & $N_{\text {samp }}$ & $N_{\text {puls }}$ & $\begin{array}{r}t_{\mathrm{s}} \\
(\mathrm{s})\end{array}$ \\
\hline $0823+26$ & 0.531 & 10.0 & 0.4 & 100 & 6200 & 925 \\
$1929+10$ & 0.227 & 10.0 & 0.2 & 200 & 3472 & 1900 \\
$1933+16$ & 0.359 & 1.0 & 0.2 & 450 & 2403 & 45 \\
\hline
\end{tabular}

Pulsars PSR $1929+10$ and PSR $1933+16$ both have triple profiles with a strong central 'core' component and weaker 'conal' outriders.

We have extracted intensities for the components of each pulse, integrating over the relevant window of pulse phase. Noise regions on the baseline well away from the pulse were also measured for comparison. To exclude extrinsic variation due to diffractive scintillation, the pulse intensities were demodulated by a running mean over the scintillation timescale $t_{s}$, as calculated from the values tabulated in Cordes (1986) and listed in table 1, and normalized to an rms intensity of unity.

We examined the autocorrelation function of the components to determine characteristic timescales for the pulse to pulse variability. All three components of PSR 1929+10 have autocorrelation functions with only power at zero lag, suggesting that the only significant fluctuations are purely stochastic. For PSR $1933+16$ again the three autocorrelation spectra were similar, showing significant correlated power out to lags of $\sim 3-4$ pulses. PSR $0823+26$ showed unusual structure, with power oscillating from positive to negative on a timescale of $\sim 2-3$ pulses.

The light curves for the various pulse components form a one dimensional record of the modulation process. However, if more than one variable controls the intensity variations, the signature of these dependencies can be recovered by sequentially differentiating or lagging the data to produce a multidimensional vector at each time step (Packard et al. 1980). The trajectory of the data set in this multi-dimensional phase space may be analyzed for structure. If, for example, the points accumulate on a strange attractor, a fractal manifold of nonintegral dimension, this is a signature of deterministic chaos.

The first such step beyond spectral methods for characterizing the fluctuations is to produce the 'phase portrait', by plotting component intensities against the flux of the following pulse. As expected, the phase portraits of PSR $1929+10$ components show a featureless collection of points about the mean pulse intensity, indicative of a dominating stochastic noise process. PSR $1933+16$ components 1 and 2, despite showing autocorrelation structure display a fairly uniform blob of points slightly elongated along the line $x=y$. This elongation is the signature of slow intensity variations and is indeed even more prominent when the slow extrinsic modulation due to interstellar scintillation is not removed. For the weak conal component 3 there seems to be slight tendency for points to extend horizontally at relatively strong $I_{n+1}$ and weak $I_{n}$.

For PSR $0823+16$ a large scatter in the points is present, but there is substantial underlying structure in the phase portrait, reproduced in figure 1. Two features draw particular attention. First,

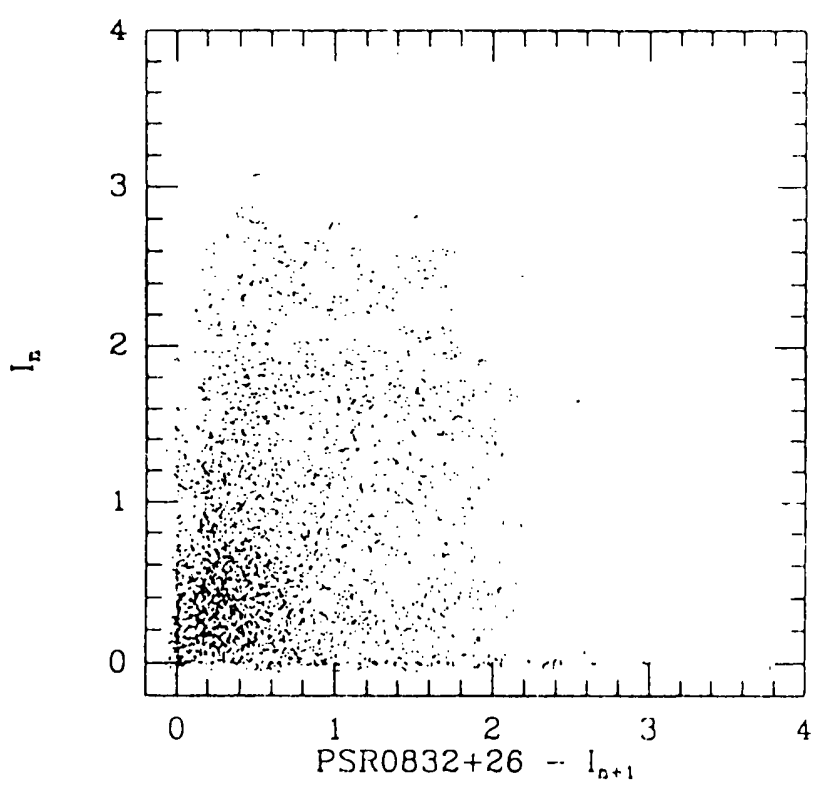

Figure 1 Phase portrait from 6200 pulses of PSR 0823+26 (see text).

for points with $I_{n}>1$, there is a significant cluster along the $x$-axis $\left(I_{n+1} \sim 0\right)$. Moreover, very faint pulses are typically preceeded by pulses of subaverage flux. This is a clear signature of pulse nulling over $\sim 2-3$ periods. In PSR $0823+26$, nulls are followed by strong pulses; similar behavior evidently might account for the weak structure in PSR 1933+16's component 3 phase portrait. In addition, at intensities $\sim 0.1-0.5$ of the rms flux, points are seen to cluster in crescent shaped region, avoiding the line $x=y$.

Such structure suggests that there is an embedded attractor and by extending the analysis to higher phase space dimensions it is possible to mitigate to some extent the effect of the obscuring stochastic noise. The fractional dimension of the manifold on which the strange attractor lives, if of dimension $d$, will be fully contained in a phase space of dimension $n>d$. Processes described by periodic behavior will be confined to manifolds of integral dimension. A stochastic process will produce random values without any temporal correlation, so that the $n$-dimensional vector formed from such a 
time series will range freely throughout phase space. Therefore deterministic processes, chaotic and periodic, can be identified by noting that orbits lie in a subset of phase space of dimension $d<n$. To measure such a region we compute the "correlation exponent", $\nu$, where the number of points within a $n$-ball of radius $r$ is $C(r) \sim r^{\nu}$. We estimate

$$
C(r)=\lim _{N \rightarrow \infty} \frac{1}{N(N-1)} \sum_{i, j ; i \neq j} \Theta\left(r-\left|\mathbf{X}_{i}-\mathbf{X}_{j}\right|\right),
$$

where $\theta$ is the Heaviside step function. Following Lochner et al., we take the distance between the phase space points $\mathbf{X}_{\mathbf{i}}$ to be given by the maximum component separation (the $L_{\infty}$ norm) and compute $C(r)$ for a range of embedding dimensions $n$. Data streams from each pulse component were analyzed for dimensions $n=1-10$. To calibrate the expected behavior we also analyzed results from a random white noise time series, mixed in varying proportions with values computed for a well known low dimensional strange attractor, the Henon-Heiles object given by the map $\left(x_{i+1}=y_{i}+1-1.4 x_{i}^{2}, y_{i+1}=0.3 x_{i}\right)$ which has dimension $d \approx 1.26$.

Results for PSR $1929+10$ and PSR $1933+16$, followed the behavior expected from random white noise quite closely over nearly two orders of magnitude in the subpulse intensity. For PSR $0823+26$ however, we show in figure 2 the exponent of $C(r)$, estimated from the first differences $\Delta \log [C(r)] / \Delta \log [r]$ with embedding dimension $n=1$ to 10 . For comparison values for a pure white noise simulation with the same number of data points are shown by the solid lines for $d=1$ to 5 . For small $r$ the scatter due to Poisson errors from finite $N_{\mathrm{p}}$ are quite apparent. In addition, the growth of $\nu$ towards $d$ is the effect of random fluctuations with an amplitude comparable to the pulse flux. However for $0.1 \gtrsim \log (r) \gtrsim-0.5$, there is a definite departure of $\nu$ from noise-like behavior, before the random fluctuations dominate at small $r$. Although inadequate scaling range in the the low noise region prevents the slopes from converging to a stable value as $n$ is increased, the presence of significant departures by $n=2$ suggests a dimension $d \lesssim 2$. Additional structure for the very brightest pulses at large $r$ is presumably related to the nulling behavior noted above.

\section{Conclusions}

We have found tentative evidence for deterministic chaos in the intensity fluctuations of PSR $0823+26$.

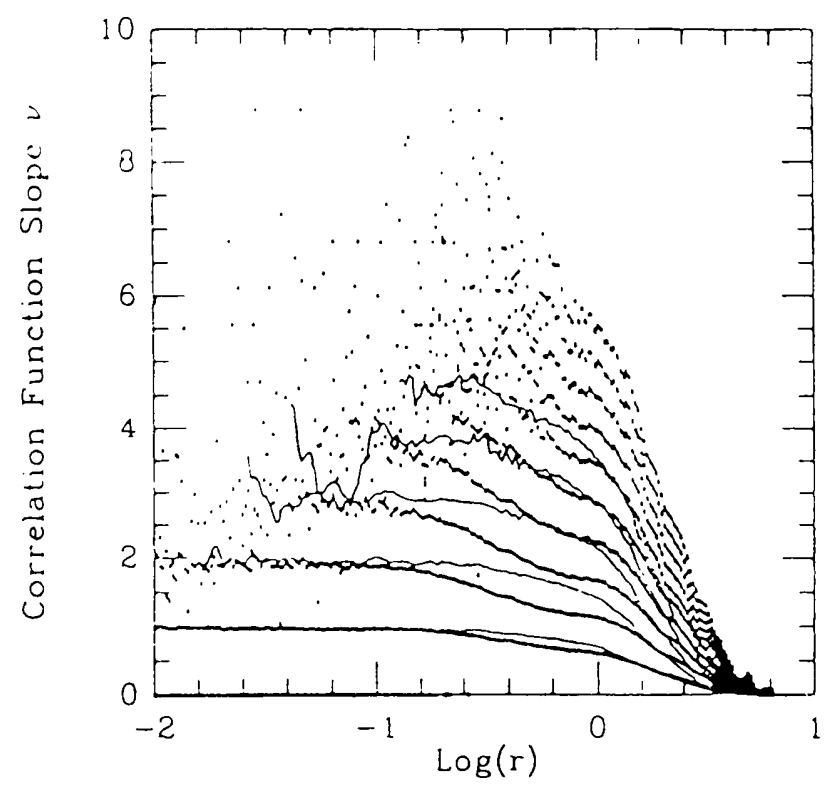

Figure 2 Slope $\nu$ of the correlation integral as a function of intensity variation $r$ for embedding dimensions $n=$ 1 to 10 . The behavior of white noise points follow the solid lines $n=1$ to 5 .

The absence of such behavior in the strong core emission from PSR 1929+10 and PSR 1933+16 and possible structure in the best separated outrider pulse component examined, suggest such behavior, like drifting subpulses, is a signature of conal emission. This interpretation receives additional credence if our identification of nulling activity in PSR $0823+26$ allows us to reassign this component to the class of conal single $\left(S_{d}\right)$ emission. This nulling activity suggests that the magnetosphere can store particles or energy for several pulse periods. The chaotic structure noted appears to be of low dimension, suggesting that only $\sim$ two coupled differential equations are required to model the fluctuations. Quasi-periodic behavior, such as drifting subpulse activity may be aligned with such low dimensionality. To strengthen the observation, analysis of a larger number of pulses and the selection of pulses with lower noise strength is required. Observation during bright scintillations can increase the signal to noise at the receiver, but apparently much of the fluctuation here is due to a noise component intrinsic to the pulsar itself. Examination of other pulsars with coherent subpulse structure would seem a profitable arena for the search for other low $d$ attractors. 